BULL. AUSTRAL. MATH. SOC.

$08 B 20,16 A 03,17 B 45,20605$

VOL. $23(1981), 477-478$.

\title{
MODULE STRUCTURE IN CERTAIN ALGEBRAS
}

\author{
Michael G, SCHOONEVELdT
}

For ages now, the literature has abounded with various graded algebras whose homogeneous components can be treated as modules for the general linear group (by algebra automorphisms) and the general linear Lie algebra (by derivations). Most of these algebras are relatively free (for example, polynomial algebras) but the exterior algebra of a vector space instances one which is not. This thesis is an attempt to treat these algebras in a uniform manner, with particular emphasis on the module structure of their components.

Aside from preliminaries, the thesis falls into three parts. The first gives an abstract definition of the relevant algebras; this involves a mild generalization of some concepts from Universal Algebra. The second introduces the two actions above, but treats them independently of each other. The final part brings the actions together by the process of Chevalley reduction; here, the components are treated as modules for certain distinguished subalgebras (first studied by J.E. Humphreys) of Kostant's algebra. No attempt is made in this third part to work in the framework of algebraic groups over algebraically closed fields; the actual treatment, a little primitive and unpopular, is perhaps more widely accessible to group theorists at large.

The thesis examines the problem of determining the composition factors of the components. It also gives a determination of the submodule lattice of the rank 2 polynomial algebra (in some respects an improvement on the work of Carter and Cline) and makes some progress with decomposing the

Received 6 March 1981. Thesis submitted to the Australian National University, June 1980. Degree approved: February 1981. Supervisor: Dr L.G. Kovács. 
components of the free associative, Lie and special Jordan algebras in finite characteristic. 